Supporting Information for:

\title{
Temporal Evolution of Surface Contamination Under Ultra-High Vacuum
}

Zhen Liu, ${ }^{1}$ Youngsup Song, ${ }^{2}$ Anoop Rajappan, ${ }^{1}$ Evelyn N. Wang, ${ }^{2}$ Daniel J. Preston ${ }^{1, *}$

${ }^{1}$ Department of Mechanical Engineering, Rice University, 6100 Main St., Houston, TX 77006

${ }^{2}$ Department of Mechanical Engineering, Massachusetts Institute of Technology, 77

Massachusetts Ave., Cambridge, MA 02139

*To whom correspondence should be addressed: djp@ rice.edu 


\section{S1. Preparation and characterization of gold samples}

The gold sputtering process to fabricate the samples studied in the main text was conducted using an AJA ATC Orion Sputtering System at Rice University. Atomic force microscopy images were collected using tapping mode with aluminum-coated silicon tips resonating at $320 \mathrm{kHz}$.

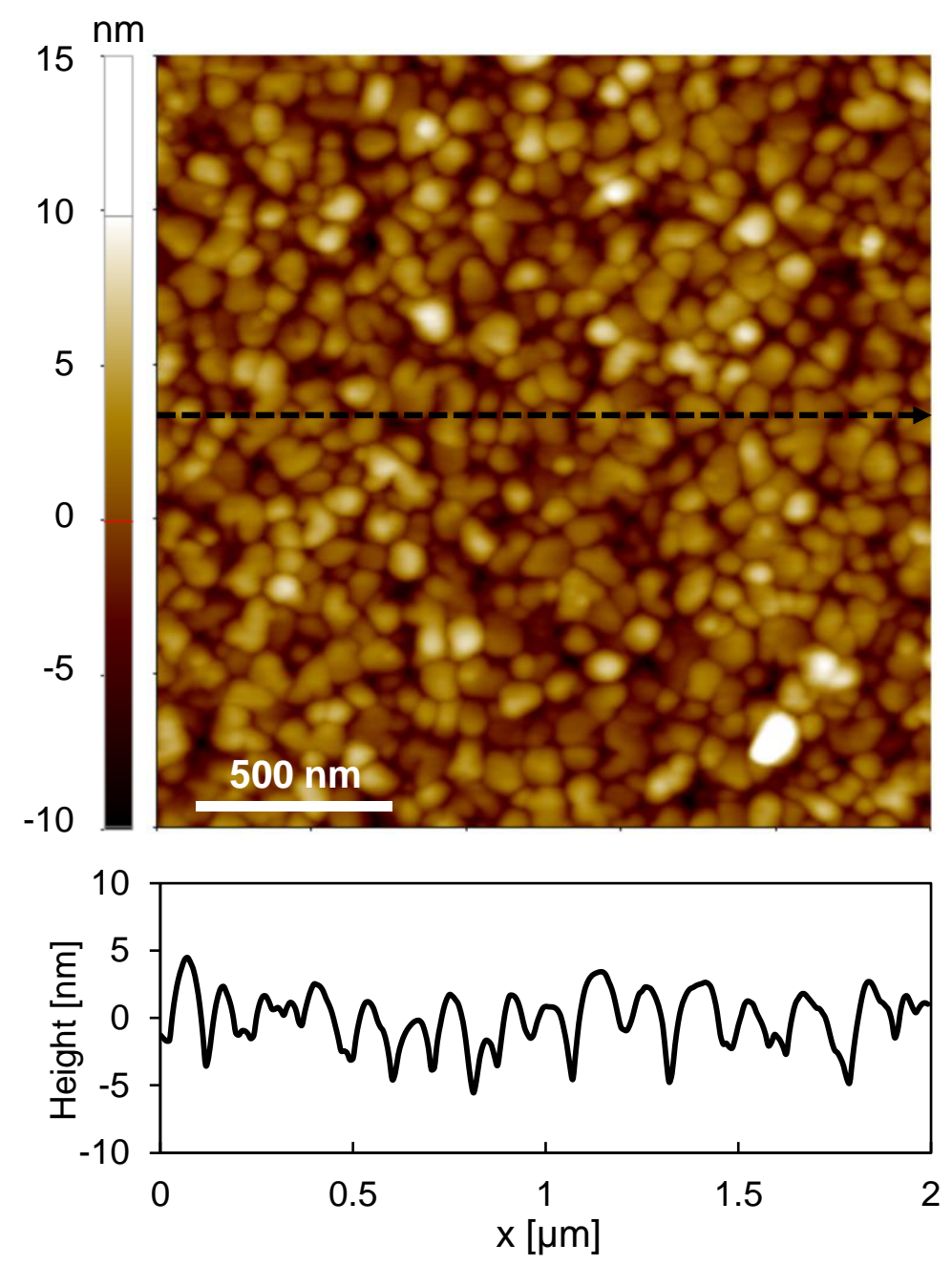

Figure S1. Atomic force microscopy scan of the gold surface (top panel) with height profile along the dashed line on the scan shown below. 


\section{S2. Surface oxygen evolution under UHV}

The in situ cleaned sample exhibited a slight increase in oxygen percentage (within 2\%) over time under UHV, potentially coming from adsorbed hydrocarbons containing oxygen molecules (e.g., carboxylic acids). Contaminated samples exhibited a decreasing level of surface concentration of oxygen over time. Because gold does not have a stable corresponding oxide, ${ }^{1,2}$ the oxygen element on the surface mainly results from the adsorbed hydrocarbons, water molecules, and the unstable gold oxide. The decrease of oxygen on contaminated samples may be due to the desorption of both hydrocarbons and water molecules, as well as the dissociation of unstable gold oxide. ${ }^{1}$
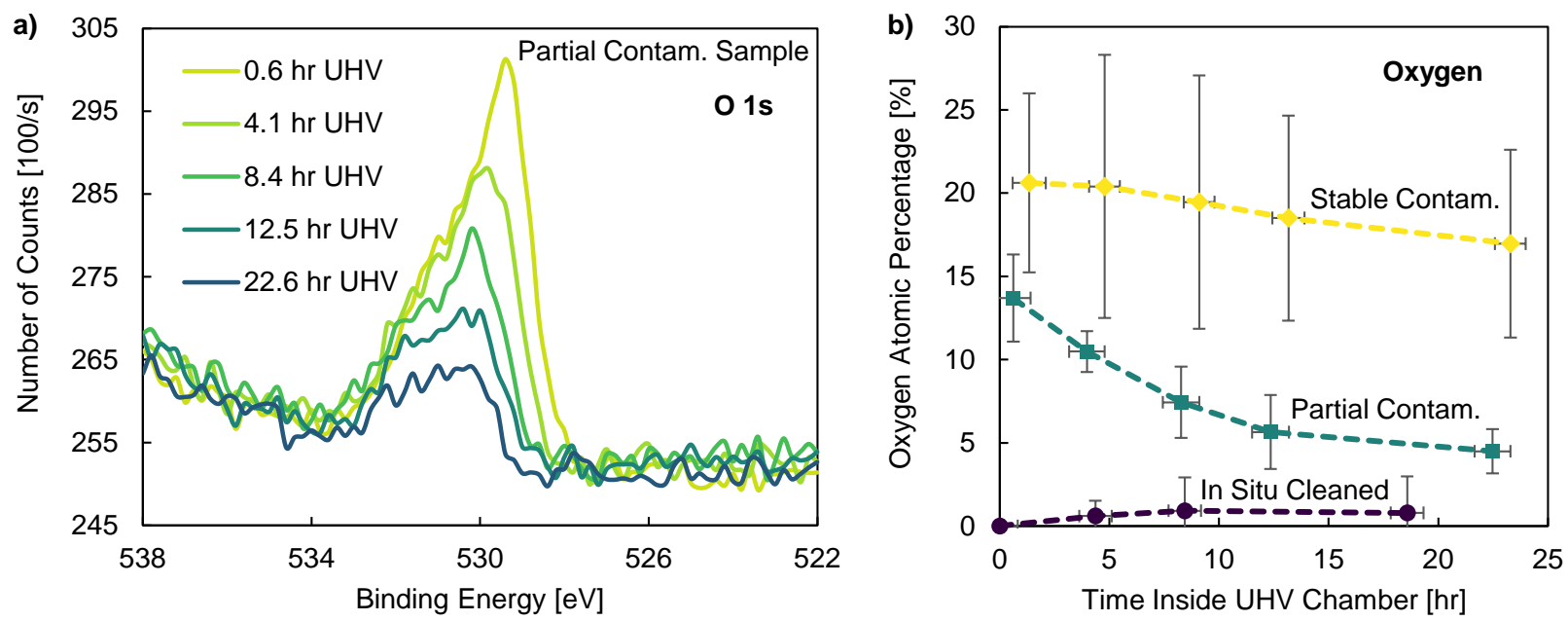

Figure S2. (a) XPS spectra for $O$ 1s of the partial contaminated sample; (b) Atomic percentage of oxygen for three samples.

\section{S3. Modeling adsorption in a gaseous atmosphere}

Hydrocarbon adsorption and desorption are reversible processes (schematic shown in Figure S3a). The surface hydrocarbon concentration reaches a dynamic equilibrium when the adsorption rate equals the desorption rate. ${ }^{3}$ Based on the Langmuir adsorption kinetic model ${ }^{4}$ and first order 
desorption theory, ${ }^{5}$ the variation of hydrocarbon surface concentration with time can be predicted by Eq. S1:

$$
\frac{d \varphi_{s}}{d t}=\frac{P}{\sqrt{2 \pi m k_{B} T}} \cdot S-v_{d} \cdot e^{\frac{\Delta H}{k_{B} T}} \cdot \varphi_{S}
$$

where $\varphi_{s}$ is the hydrocarbon concentration on the solid surface, with units of number of molecules per area. The first term on the right-hand side of the equation represents the adsorption rate, which is a function of hydrocarbon partial pressure, $P$ (determined by the volumetric hydrocarbon concentration in the surrounding ambient condition), mass of one hydrocarbon molecule, $m$, Boltzmann constant, $k_{B}$, ambient temperature, $T$, and sticking probability, $S$. The second term on the right-hand side represents the desorption rate, which is related to the surface hydrocarbon concentration, $\varphi_{s}$, the "attempt frequency," $v_{d}$ (which represents how often an adsorbed molecule attempts to overcome its potential barrier to desorption), the activation energy of desorption, $\Delta H$, and the temperature, $T$.

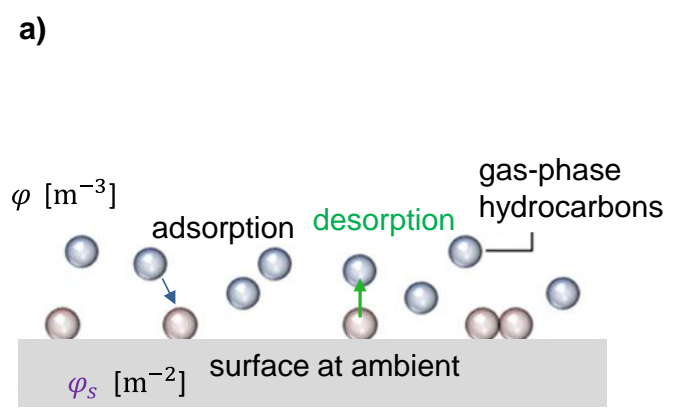

Side view of the surface b) pristine atom or hydrocarbon molecule molecule on the

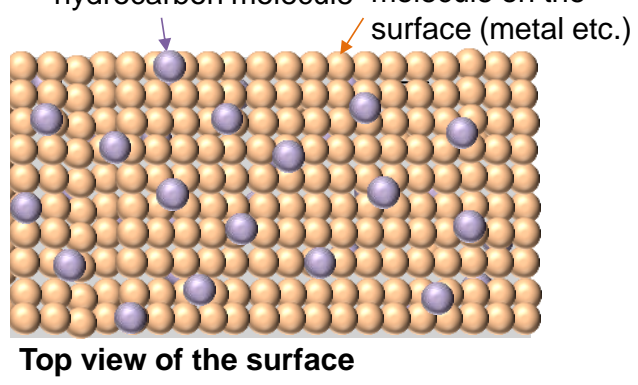

Figure S3. Schematics showing (a) the dynamic adsorption and desorption processes of hydrocarbons onto and off of the surface and (b) hydrocarbon molecules represented as defects on a pristine substrate surface. 
Assuming that the solid surfaces are exposed to the ambient atmosphere with constant temperature $T$ and constant hydrocarbon concentration, for a certain surface material, the adsorption rate from the previous equation will be constant and the desorption rate will only vary with surface hydrocarbon concentration $\varphi_{s}$. Here we define the following parameters in Eq. S2:

$$
A=\frac{P}{\sqrt{2 \pi m k_{B} T}} \cdot S \quad \text { and } B=v_{d} \cdot e^{\frac{\Delta H}{k_{B} T}}
$$

where $A$ represents the adsorption rate, $B$ is the desorption coefficient, and $B \cdot \varphi_{s}$ represents the desorption rate. $A$ and $B$ remain constant under our assumptions.

The values of $A$ and $B$ are difficult to determine because $P, S, v_{d}$, and $\Delta H$ remain poorly characterized for most materials. To obtain the estimated values for $A$ and $B$, we fitted the parameters from previous experimental data provided in the literature ${ }^{6}$ for gold, ceria, holmia, and silica, which include a metal, metal oxides, and a nonmetal oxide. The experimental data are provided in surface atomic percent carbon, $\% C$, as a function of time exposed to laboratory air after argon ion bombardment surface cleaning ${ }^{7}$ at the instant of $t=0$. In order to get fitting parameters from the experimental data, the atomic percent carbon (number of carbon atoms per number of total atoms) must be converted to hydrocarbon concentration on the surface (number of hydrocarbon molecules per area). As Figure S3b shows, we assumed the hydrocarbon adsorption phenomenon as hydrophobic defects on an initially hydrophilic surface, and the hydrocarbons as circular defects on the surface. ${ }^{8}$ Thus, the atomic carbon percent can be expressed as (Eq. S3):

$$
\% C=\frac{n \cdot N_{H C}}{n \cdot N_{H C}+m \cdot N_{M O X}} 100 \%
$$

where $N$ is the number of molecules; $n$ represents the number of carbons in one hydrocarbon 
molecule, then $n \cdot N_{H C}$ is the number of carbon atoms; likewise, $m$ is the number of atoms in one molecule or atom of the pristine surface, then $m \cdot N_{M O X}$ is the number of atoms in the metal oxide molecules. To obtain the hydrocarbon concentration on the surface, the projected areas of each hydrocarbon molecule and pristine molecule or atom on the surface are required. This area, termed "Area," could be approximated from the molar specific volume $V$ and a spherical molecule assumption. Finally, the hydrocarbon concentration on the surface is calculated as (Eq. S4):

$$
\varphi_{S}=\frac{N_{H C}}{\operatorname{Area}_{H C} \cdot N_{H C}+\operatorname{Area}_{M O X} \cdot N_{M O X}}=\frac{1}{\operatorname{Area}_{H C}+\operatorname{Area}_{M O X} \cdot \frac{N_{M O X}}{N_{H C}}}
$$

The number of atoms in one molecule or atom of the pristine surface here can be obtained directly by the stoichiometric expression of the chemical formula, or calculated by the surface element analysis of the pristine surfaces from techniques such as X-ray photoelectron spectroscopy (XPS). ${ }^{9}$ As for the type of hydrocarbons, researchers have shown that there are many kinds of relevant hydrocarbons, including toluene, tetrachloroethene, methane, benzene, nonane, etc., from motor and pump oils, solvent vapors, desorbed species of plastics, and more. ${ }^{10-14}$ To simplify the model, we use hexane $(n=6)$ to represent all the hydrocarbons in the atmosphere.

The modeling results for gold, ceria, holmia, and silica are shown in Figure S4; meanwhile, the fitting values of $A$ and $B$ are listed in Table S1. As shown in Figure S4, the final stable hydrocarbon concentrations achieved for these four different materials possess positive correlations with the advancing water contact angle from the previous experiments (as shown in Table S1), ${ }^{6}$ indicating that our assumption for the conversion from atomic percent carbon to surface hydrocarbon concentration is reasonable; the curves obtained from the adsorption-desorption model match well with the hydrocarbon concentration derived from the experimental data, which illustrates that our model is plausible. 


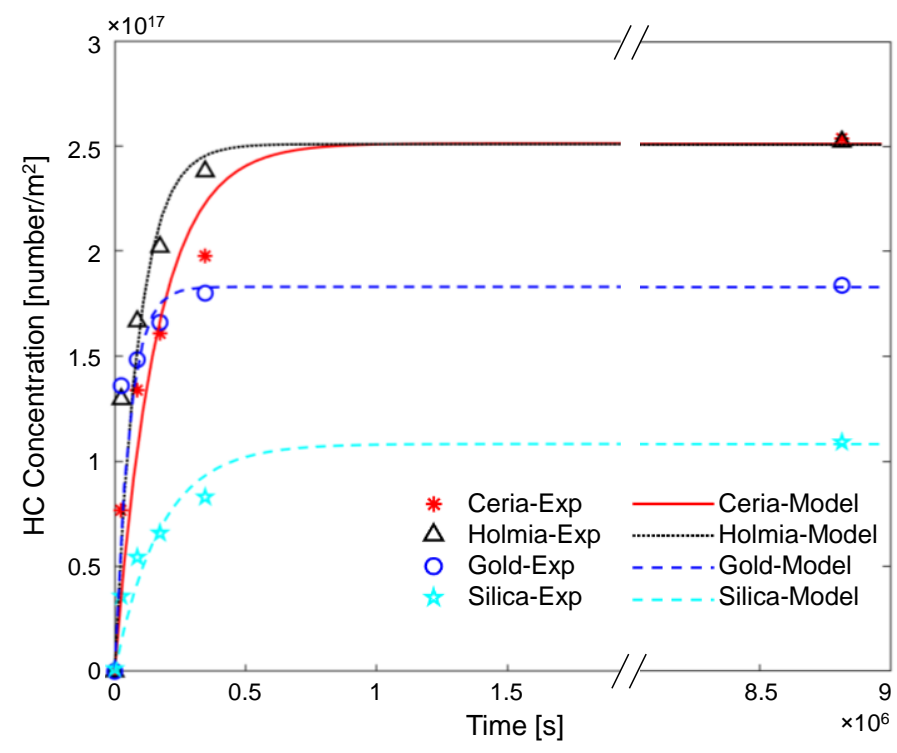

Figure S4. Surface hydrocarbon concentration as a function of time for ceria, holmia, gold, and silica exposed to laboratory air (temperature $\approx 25^{\circ} \mathrm{C}$ and relative humidity $\approx 35 \%$ ) with argon ion bombardment surface cleaning at the instant of $t=0$. The discrete points are converted from the previous experimental data, ${ }^{6}$ the continuous lines depict the modeling results.

Table S1. Fitting parameters of the model and advancing water contact angle (WCA) after long exposure time (2448 hours) from experiments for ceria, holmia, gold, and silica. The bolded quantities are fitting values for gold in ambient air condition, as being compared with the parameters under UHV.

\begin{tabular}{lccc}
\hline \multirow{2}{*}{ Sample } & Adsorption rate & Desorption coefficient & WCA [deg.] \\
& $A\left[\mathrm{~m}^{-2} \cdot \mathrm{s}^{-1}\right]$ & $B\left[\mathrm{~s}^{-1}\right]$ & \\
\hline Ceria $\left(\mathrm{CeO}_{2}\right)$ & $15.83 \times 10^{11}$ & $6.3 \times 10^{-6}$ & $94.77 \pm 6.76$ \\
Holmia $\left(\mathrm{Ho}_{2} \mathrm{O}_{3}\right)$ & $27.7 \times 10^{11}$ & $11.04 \times 10^{-6}$ & $103.06 \pm 6.08$ \\
Gold $(\mathrm{Au})$ & $\mathbf{5 . 2 6} \times \mathbf{1 0}^{\mathbf{1 2}}$ & $\mathbf{2 . 8 9} \times \mathbf{1 0}^{-5}$ & $65.99 \pm 3.92$ \\
Silica $\left(\mathrm{SiO}_{2}\right)$ & $6.18 \times 10^{11}$ & $5.71 \times 10^{-6}$ & $43.95 \pm 4.04$ \\
\hline
\end{tabular}




\section{S4. Timescale of desorption for the stable contaminated sample under UHV}

The initial stronger bonding between the adsorbent and gold surface is a certain finite number of molecules. It would decrease over time as more and more of the newly adsorbed hydrocarbons on the surface exhibit weaker bonding. If the desorption process of those initial stronger bonding is too fast or too slow compared with the experimental time, the variation of desorption coefficient $B$ can be neglected. Figure S5 shows that the timescale to remove $99 \%$ of the initial hydrocarbons is around 24 hours, which is comparable to our experiment period, indicating that the variation of desorption coefficient of the stable contaminated sample is non-negligible.

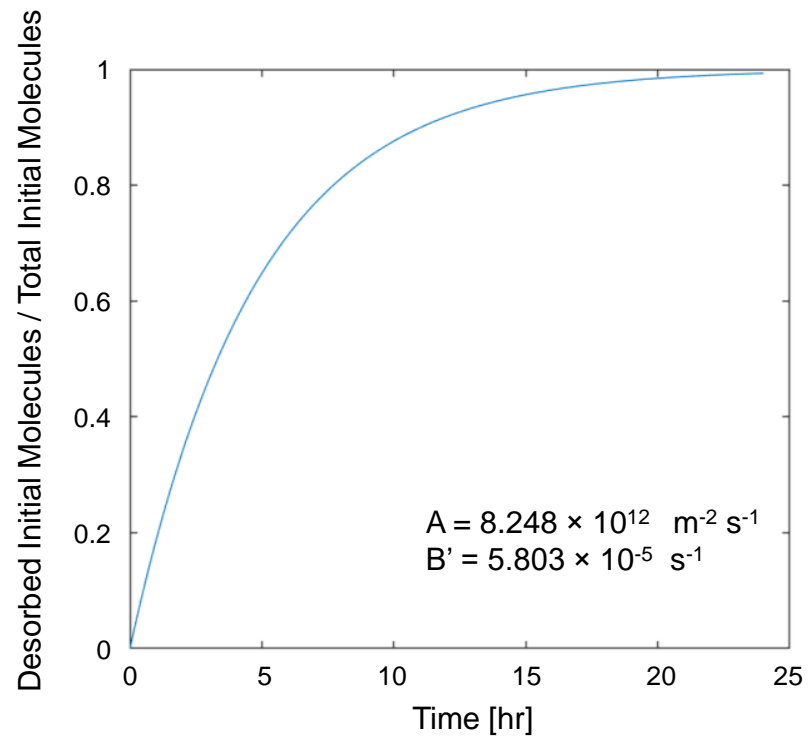

Figure S5. The proportion of desorbed initial (more stably bonded) molecules to the initial quantity of molecules for the stable contaminated sample. 


\section{S5. Atomic carbon percentage on XPS stage platen}

To identify a source of contamination in our work, we characterized the surface elemental composition of the sample stage platen (which is removed from the system via a load lock device and exposed to the laboratory air while mounting samples for analysis, as shown in the bottom inset picture in Figure S6) of the XPS system at Rice University. We selected five individual measuring points on the platen, and the average value was reported. Figure S6 shows that the atomic carbon percentage of the platen is over $70 \%$ even after 6 hours under UHV, indicating that the platen could be a significant source of hydrocarbon contaminants in the XPS chamber.

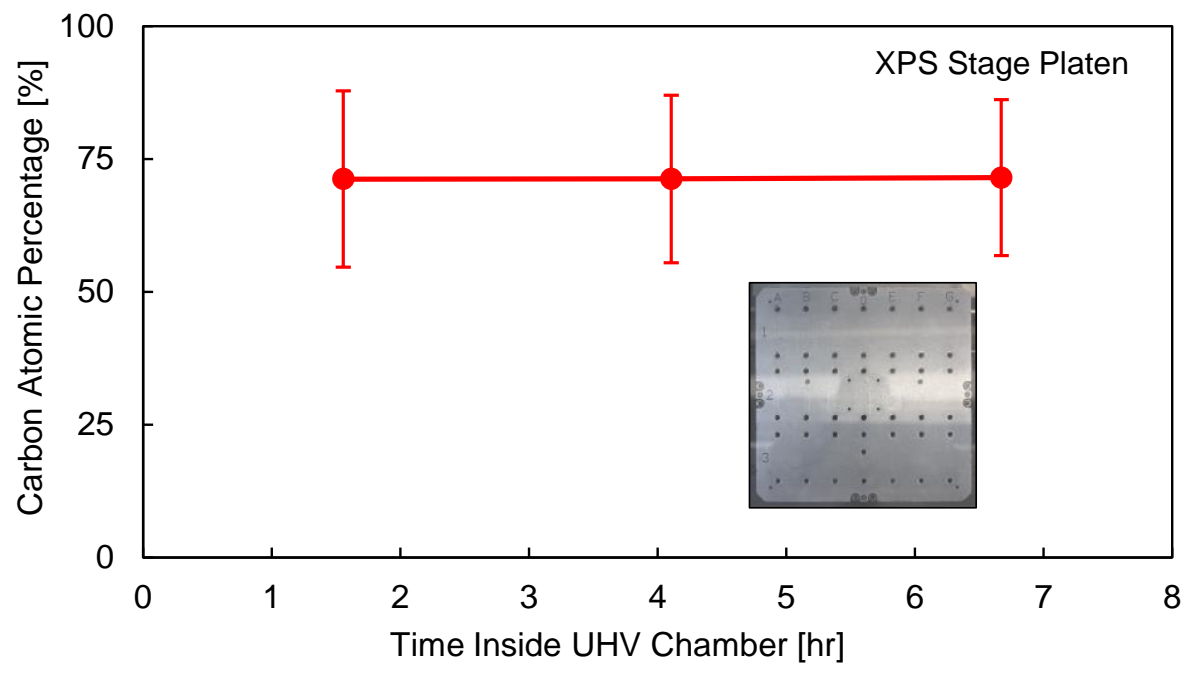

Figure S6. Atomic carbon percentage on the stage platen used to mount samples in the XPS chamber. A photo of the platen is shown in the bottom inset picture. 


\section{SUPPORTING INFORMATION REFERENCES}

1. Tsai H, Hu E, Perng K, Chen M, Wu JC, Chang YS. Instability of gold oxide Au2O3. Surf Sci. 2003;537(1-3). doi:10.1016/S0039-6028(03)00640-X

2. Jones PG, Rumpel H, Schwarzmann E, Sheldrick GM, Paulus H. Gold (III) oxide. Acta Crystallogr Sect B Struct Crystallogr Cryst Chem. 1979;35(6):1435-1437.

3. Somorjai GA, Mujumdar AS. Introduction to surface chemistry and catalysis. Dry

Technol. 1995. doi:10.1080/07373939508916972

4. Langmuir I. The adsorption of gases on plane surfaces of glass, mica and platinum. $J$ Am Chem Soc. 1918;40(9):1361-1403. doi:10.1021/ja02242a004

5. Redhead PA. Thermal desorption of gases. Vacuum. 1962. doi:10.1016/0042207X(62)90978-8

6. Preston DJ, Miljkovic N, Sack J, Enright R, Queeney J, Wang EN. Effect of hydrocarbon adsorption on the wettability of rare earth oxide ceramics. Appl Phys Lett. 2014;105(1):18. doi:10.1063/1.4886410

7. Raiber K, Terfort A, Benndorf C, Krings N, Strehblow HH. Removal of self-assembled monolayers of alkanethiolates on gold by plasma cleaning. Surf Sci. 2005;595(1-3):56-63. doi:10.1016/j.susc.2005.07.038

8. Raj R, Enright R, Zhu Y, Adera S, Wang EN. Unified model for contact angle hysteresis on heterogeneous and superhydrophobic surfaces. Langmuir. 2012;28(45):15777-15788. doi:10.1021/la303070s

9. Briggs D. X-ray photoelectron spectroscopy (XPS). Handb Adhes Second Ed. 2005:621622. doi:10.1002/0470014229.ch22

10. Ito A, Takahashi I, Nagata Y, Chiba K, Haraguchi H. Spatial and temporal characteristics 
of urban atmospheric methane in Nagoya City, Japan: An assessment of the contribution from regional landfills. Atmos Environ. 2001;35(18):3137-3144. doi:10.1016/S13522310(00)00533-1

11. Lee SC, Chiu MY, Ho KF, Zou SC, Wang X. Volatile organic compounds (VOCs) in urban atmosphere of Hong Kong. Chemosphere. 2002;48(3):375-382. doi:10.1016/S00456535(02)00040-1

12. Liu Y, Shao M, Fu L, Lu S, Zeng L, Tang D. Source profiles of volatile organic compounds (VOCs) measured in China: Part I. Atmos Environ. 2008;42(25):6247-6260. doi:10.1016/j.atmosenv.2008.01.070

13. Parra MA, Elustondo D, Bermejo R, Santamaría JM. Ambient air levels of volatile organic compounds (VOC) and nitrogen dioxide (NO2) in a medium size city in Northern Spain. Sci Total Environ. 2009;407(3):999-1009. doi:10.1016/j.scitotenv.2008.10.032

14. Kamal MS, Razzak SA, Hossain MM. Catalytic oxidation of volatile organic compounds (VOCs) - A review. Atmos Environ. 2016;140:117-134.

doi:10.1016/j.atmosenv.2016.05.031 